\title{
Instability Issues at the SNS Storage Ring*
}

\author{
S.Y. Zhang \\ Broothaven National Laboratory, Upton, NY 11973
}

\begin{abstract}
The impedance and beam instability issues of the SNS storage ring is reviewed, and the effort toward solutions at the BNL is reported. Some unsettled issues will be raised, indicating the direction of planned works.
\end{abstract}

\section{SNS Storage Ring}

The parameters of the SNS storage ring are listed in Table 1. Several relevant issues in the machine design are as follows.

1 With the very high intensity of $2.08 \times 10^{14}$ protons per pulse, the space charge effect and the particle distribution in transverse phase space need attentions [1].

2 At $2 M W W$, the beam power of the SNS is about 12 times higher than the ISIS, and about 25 times higher than the PSR. In the 1996 survey, with 4.5 hours cooldown after the operations at about $56 \mathrm{KW}$ level, there are still 31 contact readings at the ends of sections show the activation higher than $2 \mathrm{rem} / \mathrm{hr}, 11$ of them higher than $5 \mathrm{rem} / \mathrm{hr}$ [2]. This activation affects operations at the PSR For the SNS, therefore, the uncontrolled beam loss is not allowed to exceed $0.02 \%$ of the beam intensity. One approach to achieve this goal is to use large machine aperture, which then has impact on the impedance and instabilities.

\begin{tabular}{|c|c|c|c|}
\hline Parameter & & SNS & Unit \\
\hline Beam Power & $P$ & 2 & $M W W$ \\
\hline Total Particle & $N$ & 2.08 & $10^{14}$ \\
\hline Circumference & $C$ & 220 & $m$ \\
\hline Kinetic Energy & $E_{k}$ & 1.0 & $G e V$ \\
\hline Repetition Rate & & 60 & $H z$ \\
\hline Bunch Length & $t_{B}$ & 550 & $n s$ \\
\hline Injection Turms & & 1200 & \\
\hline RF Voltage, $h=1 / 2$ & $V_{R F}$ & $40 / 20$ & $K V$ \\
\hline Beam Momemtum Spread & $\Delta p / p$ & 0.7 & $\%$ \\
\hline Beam Current & $I_{0} / I_{p}$ & $40 / 80$ & $A$ \\
\hline Ave. Chamber Radius & $b$ & 10 & $\mathrm{~cm}$ \\
\hline Uncontr. Beam Loss & & 0.02 & $\%$ \\
\hline Beam Loss Power /m & & 1.8 & $W / m$ \\
\hline
\end{tabular}

Table 1: SNS storage ring parameters

CP496. Workshop on Instabilities of High Intensiry Hadron Beams in Rings. edited by $T$. Roser and S. Y. Zhang

(C) 1999 American Institute of Physics 1-56396-910-6/99/\$15.00 
3 The SNS is intentionally designed with a relatively high RF voltage. The large beam momentum spread defined during the multiturn injection, therefore, may help the damping of the longitudinal microwave instabilities. The high RF voltage also brings along with the tolerance for the longitudinal space charge effect, which is defocusing and causes the bunch leakage into the gap.

4 The bunch in the SNS storage ring is very long, however, it only has to stay in the machine for about $1 \mathrm{~ms}$. Immediately after the multiturn injection, the beam is extracted. This calls for better understanding of the impedance and instability issues during the beam stacking.

In this paper, selected impedance and instability issues will be discussed. Also, unsettled issues will be raised, indicating the directions of the planned works.

\section{Impedance}

The impedances of the SNS storage ring, like other synchrotrons, fall into several categories as follows.

1. Space charge impedance is frequency independent. These are calculated for the SNS storage ring as $Z_{\ell} / n=-j 196 \Omega$ and $Z_{T}=-j 6.87 \mathrm{M} \Omega / \mathrm{m}$, for longitudinal and transverse, respectively. This impedance is a dominant one.

2. Resistive wall impedance is of interest at very low frequency range. The relation of $Z_{T} \approx\left(Z_{\ell} / n\right) 2 R / \beta b^{2}$ exists. Sometimes, one estimates the resistive wall impedance by its value at the revolution frequency. At the SNS, the longitudinal and transverse resistive wall impedances at the revolution frequency are $Z_{\ell}\left(\omega_{0}\right)=0.65(1+j) \Omega$ and $Z_{T}\left(\omega_{0}\right)=5.22(1+j) K \Omega / m$, respectively.

3. Resonant frequency of broadband impedance is in a few $G H z$. Therefore, below the cut off frequency $1 \mathrm{GHz}$, this impedance can be seen as an inductance.

4. Resonant modes of low frequency impedance are in a few tens to several hundred $M H z$. In the relevant frequency range of up to $1 \mathrm{GHz}$, the imaginary part of the impedance cannot be seen as a pure inductance, and the real part may not be negligible. The contributors of the low frequency impedance include the BPM, the extraction and injection kickers. The impedance of the BPM of the SNS storage ring is handily calculated using conventional formulations $[3,4]$, the real part of the impedance is peaked around $100 \mathrm{MHz}$, where the longitudinal and transverse impedances are $Z_{\ell} / n \approx 2 \Omega$ and $Z_{T} \approx 40 \mathrm{~K} \Omega / \mathrm{m}$, respectively.

5. Narrow band impedances are mainly from the RF cavities. However, parasitic parameter may cause sharp resonance in the kicker impedance, and large steps and cavities of the vacuum chamber may also contribute to the narrow band impedance. 
In the design of the SNS storage ring, some concerns with respect to the broadband and narrow band impedances, and also the extraction kickers have been raised. Therefore, these impedances will be discussed separately as follows.

\section{A. Broadband Impedance}

The bellows, steps, ports, collimators, etc., contribute to the broadband impedance. The general relation between the longitudinal and transverse impedances, $Z_{T} \approx$ $\left(Z_{\ell} / n\right) 2 R / \beta b^{2}$, can be applied. In Table 2 , the broadband impedances due to each component are shown for the SNS storage ring, using the analytical calculations [5].

\begin{tabular}{|c|c|c|}
\hline & $Z_{\ell} / n$ & $Z_{\mathcal{T}}$ \\
\hline Bellows & 1.1 & 8.8 \\
\hline Vacuum ports & 0.49 & 3.9 \\
\hline Valves & 0.28 & 2.2 \\
\hline Steps & 16.8 & 134.4 \\
\hline Collimator & 1.04 & 8.3 \\
\hline Total & 19.7 & 157.6 \\
\hline Unit & $j \Omega$ & $j K \Omega / m$ \\
\hline
\end{tabular}

Table 2: Broadband impedance

The broadband impedance is dominated by large steps, typically $2 \mathrm{~cm}$. At present, only moderate tapering and shielding are proposed. Further tapering and complete shielding can reduce the broadband impedance to just a few $\Omega s$. However, not only the cost is an issue, the machine aperture is also affected in further tapering and shielding.

On the other hand, the broadband impedance calculated for the SNS storage ring is comparable with $j 14 \Omega$ for ISR [6], $j 16 \Omega$ for SPS [7], $j 30 \Omega$ for AGS [8], and $j 17$ $\Omega$ for CPS [9]. Experience in the operations of these machines did not show that the broadband impedance posed the limit of beam intensity or caused additional beam loss.

\section{B. Narrow Band Impedance}

The narrow band impedance comes mainly from the $R F$ cavity high order modes. The large steps and other cavities in the chamber also contribute to the narrow band impedance in the range around $G H z$. In addition, it will be shown that the unmatched extraction kickers also contribute to the narrow band impedance.

Coupled bunch instability, caused by the narrow band impedance, is not relevant to the SNS. On the other hand, theoretically, narrow band impedance may cause instabilities for long bunches, for instance, the transverse Robinson instability [10]. 
For the low or medium energy hadron machines, however, no evidence has been shown that this impedance has posed serious limitations.

Better understanding of the impact of the narrow band impedance is needed, prior to take possible further steps beyond the moderate tapering and shielding.

\section{Extraction Kicker, Transverse}

The extraction kicker of the SNS storage ring consists of 8 window frame magnet units, with the average length of $\bar{\ell}=40 \mathrm{~cm}$, the average width of $2 \bar{b}=14 \mathrm{~cm}$, all have height $2 a=11.5 \mathrm{~cm}$. The impedance of this kicker is not only relatively large, but also very sensitive to the terminations.

Consider the conventional calculation [11],

$$
Z_{T}=\frac{\omega \omega \mu_{0}^{2} \ell^{2}}{4 a^{2} Z_{k}} \Omega / m
$$

where $Z_{k}=j \omega L+Z_{g}$, with $L$ the magnet inductance, and $Z_{g}$ the termination impedance. For $Z_{g}=200 \Omega$, the extraction kicker transverse impedance is shown in Fig. 1, where we observe that the real part of the impedance, $Z_{T \text {,real }} \approx 20 \mathrm{~K} \Omega / \mathrm{m}$, is peaked at $30 \mathrm{MHz}$.

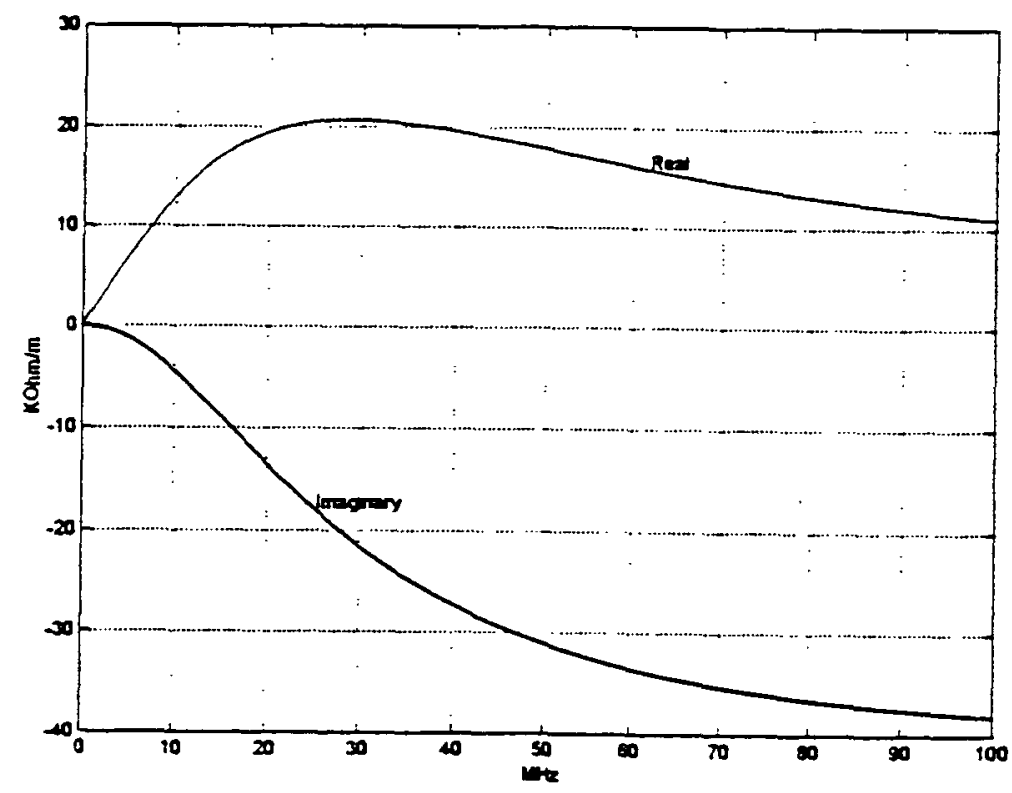

Fig. 1

Using a more realistic termination with 50 pf capacitance presented around the stray inductance of $0.5 \mu H$, the picture of the impedance is dramatically changed, as shown in Fig. 2. Two resonances with much narrower bandwidth are shown at 20 $M H z$, and $50 \mathrm{MHz}$, with the real parts of $Z_{T \text { real }} \approx 100 \mathrm{~K} \Omega / \mathrm{m}$, and $Z_{\text {T,real }} \approx 200$ 
$K \Omega / m$, respectively. Sensitivity of the impedance with respect to the termination can also be shown if the charging resistance and the stray parameters are changed.

The AGS injection kicker and the AGS Booster extraction licker have used the same structure of window frame magnets. The terminations of the magnet windings did not require special attentions. There are, however, some speculations that these kicker impedance might be responsible to certain instabilities. Therefore, the measurement of the extraction kicker impedance is undergoing in order to optimize the terminations.

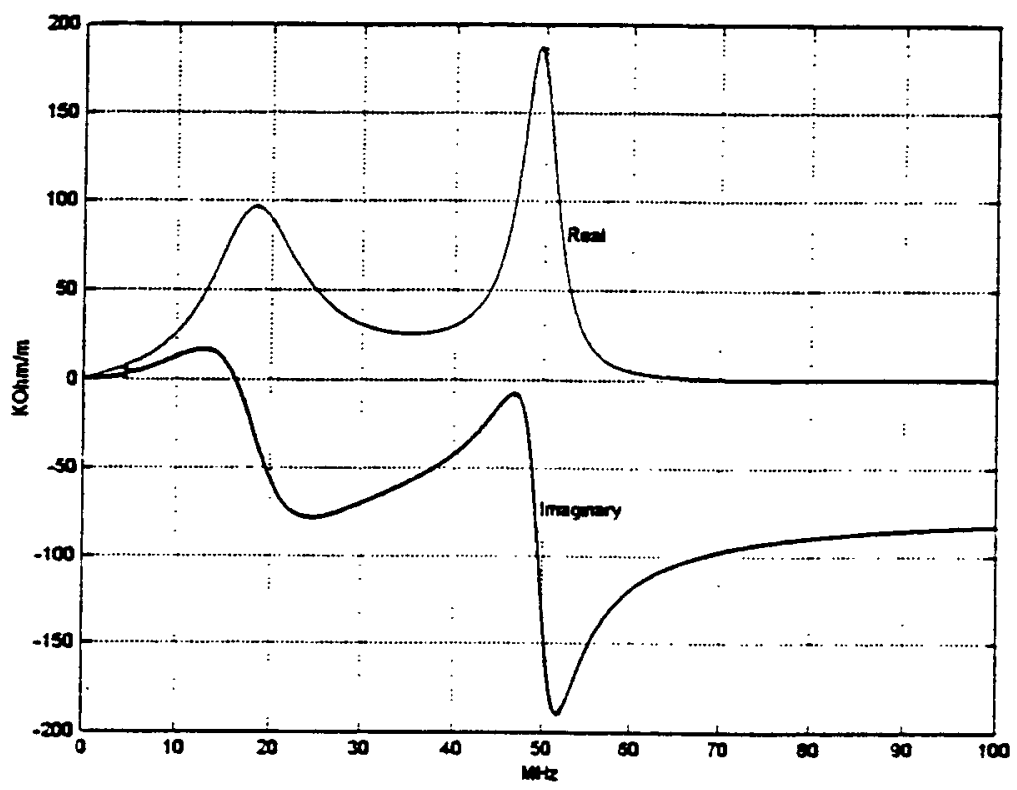

Fig. 2

\section{Extraction Kicker, Longitudinal}

To reduce the massive ferrite loss, copper sheets are placed in the ferrite core of the window frame magnet as flux break. Taking the thickness of the copper sheet as $1 \mathrm{~mm}$, the total leakage inductance around it is calculated as $L_{\text {leak }} \approx 6 \mu H$ [12]. This result is also verified by the simulation [13]. Using the formulation,

$$
\frac{Z_{\ell}}{n}=j \omega_{0} L_{\text {leak }}
$$

we get the equivalent longitudinal impedance $Z_{\ell} / n=j 45 \Omega$.

It is interesting to note that the SNS longitudinal space charge impedance is $-j 196 \Omega$, which is negative inductive. Therefore, the magnet flux leakage may compensate a part of that impedance.

Note that the beam image current at the conductor, which is used as the magnet winding, will partly offset the field created by the beam. The present plan is, however, 
to use a single power supply to drive both conductors, thus, the image current effect will be negligible.

Compensation of the longitudinal space charge impedance has been studied by inserting ferrite rings in the beam pipe, such as that at PSR [14] and KEK PS [15]. This approach is also proposed for the muon-collider proton driver [16]. If handled carefully, the ferrite window frame used for the extraction kicker may be used for the same purpose. A thinner copper sheet might be used without causing a heating problem [17], which is associated with the real part of the impedance.

Finally, a complete model should include a differential flux leakage through the gap air. Assuming this leakage to be a half of the leakage around the copper sheet, and the parasitic capacitance to be $50 \mathrm{pf}$, the longitudinal impedance is shown in Fig. 3. It can be observed that the linear inductive part is extended up to $40 \mathrm{MHz}$, beyond that the real part of impedance will take place. The effect of this real part of the impedance on the longitudinal microwave instability probably needs attentions.

Termination is also very important in determining this impedance [18], which will be determined by the measurements.

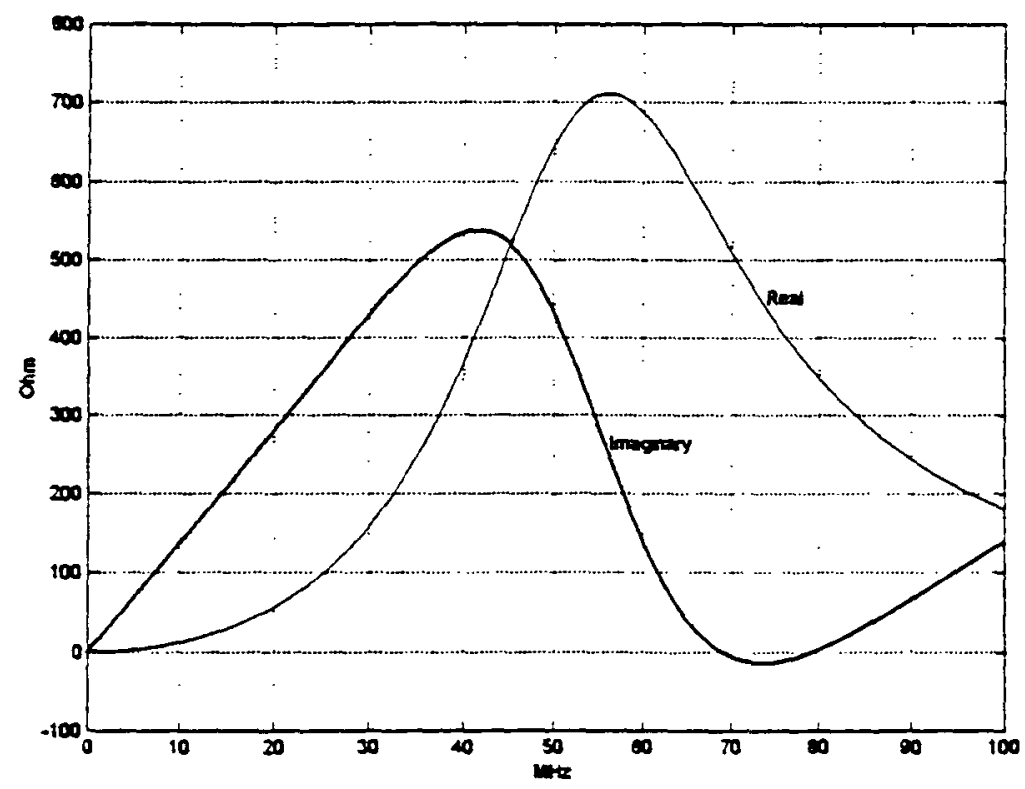

Fig. 3

\section{Instability}

The most relevant instability issues at the SNS storage ring include the longitudinal microwave, the resistive wall, and the transverse microwave instabilities. 


\section{A. Longitudinal Microwave Instability}

Taking the beam peak current of $80 \mathrm{~A}$, the Keil-Schnell criterion is satisfied for the SNS storage ring if the beam momentum spread $\Delta p / p \geq 0.65 \%$. For the machines operated below transition, this criterion is believed to be too stringent. The SNS storage RF voltage of the fundamental harmonic is $40 \mathrm{KV}$, the beam momentum spread at the end of stacking is $\Delta p / p= \pm 0.7 \%$. Therefore, the longitudinal microwave instability should not happen.
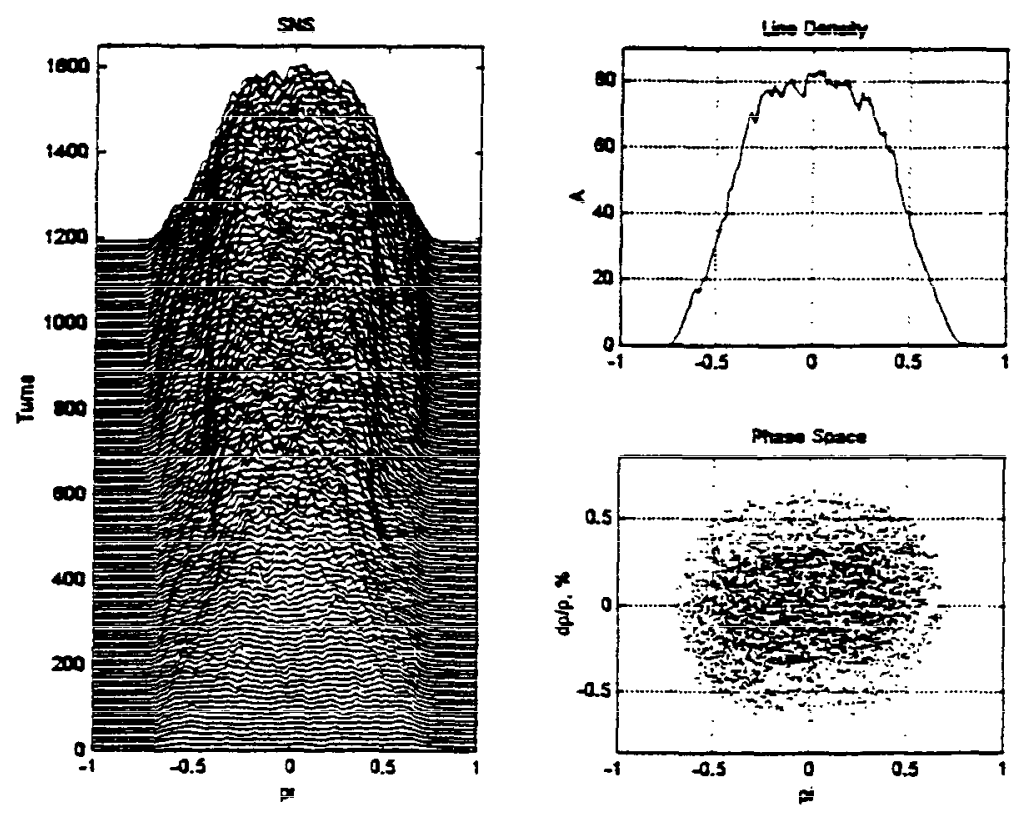

Fig. 4

One of the concerned issues in the beam stacking is the leakage of particles into the gap. The leaked proton beam is blamed for the survival of the electrons during the gap passage. The PSR study has shown that this leakage can lower the e-p instability threshold [19]. Also, for a storage ring, the leaked beam in the gap results in directly the beam loss at the extraction.

To reduce this leakage, the ramping of the RF voltage from $20 \mathrm{KV}$ at the beginning to $40 \mathrm{KV}$ at the end of stacking is proposed. The mountain range during the stacking, the line density and the particle distribution at the end of stacking are shown in Fig.4. It can be observed that the beam momentum spread has been reduced to $\Delta p / p= \pm 0.65 \%$, which is acceptable from the longitudinal microwave instability point of view.

Using the typical Linac beam momentum spread at the end of the transport line, $\Delta p / p= \pm 0.1 \%$, concerns have been raised on the beam momentum distribution in the ring. Simulations show that a large amount of particles have the momentum deviation less than $\pm 0.2 \%$, during the stacking. The effectiveness of the longitudinal microwave instability damping mechanism, based on the beam momentum spread, is questioned. 
To improve the beam momentum distribution in the ring, the Linac beam $\Delta p / p=$ $\pm 0.3 \%$ is requested, which is, in fact, used in the simulation that shown in Fig. 4. The beam momentum distribution in the ring has been improved, however, the beam loss associated with this large momentum spread probably needs attentions.

\section{B. Resistive Wall Instability}

For the stainless steel vacuum chamber used for the SNS, at the tune of $\nu=5.82$, the resistive wail instability growth rate at the end of stacking is calculated as $1 \mathrm{~ms}$ for zero chromaticity. Also, the largest growth rate of mode $m=1$ is about $1 / 5$ of the $m=0$ mode, i.e. $5 \mathrm{~ms}$, which happens at $\xi \approx-0.04$. In this calculation, damping mechanism is not included.

Using the same approach, the AGS resistive wall instability at the injection porch is calculated with the growth rate of $0.37 \mathrm{~ms}$, at the vertical tune of $\nu=8.85$. The resistive wall instability observed at the AGS has a typical growth rate of $2 \mathrm{~ms}$, which is slower than the calculated one by a factor of 5 .

Also using the same approach, the AGS Booster resistive wall instability growth rate was calculated as $0.48 \mathrm{~ms}$. In the operation, the instability has never been observed. The fast ramping at the Booster probably helps to further damp the instability.

We conclude that the resistive wall instability will not be strong enough to cause a serious problem at the SNS storage ring. Also, the choice of using stainless steel vacuum chamber is justifiable on this aspect.

\section{Transverse Microwave Instability}

\section{Transverse instability issues}

The typical bunch length at the SNS storage ring is about $550 \mathrm{~ns}$, giving rise to the full bandwidth of the bunch 3.6 $\mathrm{MHz}$. In comparison, bunch lengths of the LHC and RHIC at the storage mode are about $1 \mathrm{~ns}$, giving rise to the full bandwidth of the bunch $2 \mathrm{GHz}$.

The typical resonance of the broadband impedance is around $1 \mathrm{GHz}$. Therefore, the transverse mode coupling is of concern at both the LHC and RHIC [20]. Given the much smaller bandwidth of the bunch at the SNS storage ring, the mode coupling will not happen. Note that due to the difference between the spectrum of the modes, the mode crossing may take place, but the imaginary part will not rise.

As for the low frequency impedance, weak mode couplings may take place, but no strong mode coupling is expected. This is agreeable with the observation that no mode coupling has been causing problem in low and medium energy proton synchrotrons.

Since the entire beam life takes about a synchrotron period, conventional headtail type instability will not be a serious problem. 
The transverse microwave instability, however, may develop at a part of the long bunch, depending on the local peak current, associated impedance, and local coherent tune shift.

\section{Micrówave instability}

The rule of thumb in the transverse microwave stability is that the coherent tune shift should not exceed the incoherent tune spread. The coherent tune shift comes mainly from the space charge image effect and the broadband impedance effect. This tune shift has been frequently measured in the operated machines.

The incoherent tune spread can be written as

$$
\Delta \nu_{i n c}=\left(\left(n+\nu_{0}\right) \eta-\xi \nu_{0}\right) \frac{\Delta p}{p}+\Delta \nu_{\text {other }}
$$

where the slippage $(\eta)$ and chromatic $(\xi)$ effects are momentum dependent. Usually, $\Delta \nu_{\text {other }}$ includes the amplitude dependent octupolar tune spread and the synchrotron tune. A question often raised, is the space charge incoherent tune spread effective in damping the transverse microwave instability?

A review of the existing low and medium energy proton synchrotrons has shown that excluding the space charge incoherent tune spread, the coherent tune shifts consistently exceed the incoherent tune spread, which consists of chromatic, slippage, and synchrotron tune spread, but not the octupolar tune spread.

\begin{tabular}{|l|l|l|l|l|l|}
\hline & ISIS & AGS B & PSR & AGS & \\
\hline$N$ & 4 & 2 & 3 & 6 & $10^{13}$ \\
\hline$B_{f}$ & 1 & 0.4 & 0.4 & 0.3 & \\
\hline$E_{k}$ & 0.07 & 0.2 & 0.8 & 1.55 & $G e V$ \\
\hline$\xi$ & -1.4 & -0.2 & -0.2 & -0.2 & \\
\hline$\Delta p / p$ & 0.2 & 0.7 & 0.34 & 0.4 & $\%$ \\
\hline \hline$\Delta \nu_{\text {wall }}$ & 10.9 & 5.63 & 0.77 & 1.77 & $10^{-2}$ \\
\hline$\Delta \nu_{B B}$ & 0.73 & 0.74 & 0.35 & 1.95 & $10^{-2}$ \\
\hline$\Delta \nu_{\text {coh.total }}$ & $\mathbf{1 1 . 6 3}$ & $\mathbf{6 . 3 7}$ & $\mathbf{1 . 1 2}$ & $\mathbf{3 . 8 4}$ & $10^{-2}$ \\
\hline \hline$|\xi| \nu_{0} \Delta p / p$ & 1.09 & 0.69 & 0.14 & 0.71 & $10^{-2}$ \\
\hline$\eta \Delta p / p$ & 0.16 & 0.45 & 0.06 & 0.05 & $10^{-2}$ \\
\hline$\Delta \nu_{S}$ & 0 & 0.3 & 0.04 & 0.27 & $10^{-2}$ \\
\hline$\Delta \nu_{\text {inc.total }}$ & $\mathbf{1 . 2 5}$ & $\mathbf{1 . 4 4}$ & $\mathbf{0 . 2 4}$ & $\mathbf{1 . 0 3}$ & $10^{-2}$ \\
\hline
\end{tabular}

Table 3. Coherent and Incoherent Tunes

Consider the normal operations of PSR, AGS and its Booster at the injection energies, and also a study performed at the ISIS for coasting beam at the injection energy. The broadband impedance is assumed to be $j 30 \Omega$ for all the machines. For convenience, the mode $n=1$ is used in the calculation of the slippage tune spread. 
In Table 3, it is shown that for all these machines, the coherent tune shift is much larger than the incoherent tune spread. The gap is larger if the energy is lower.

The operations of these machines over the years, however, have not been hampered by the transverse microwave instability. It is of interest, therefore, to consider the role played by space charge tune spread in damping the transverse microwave instability.

\section{Effect of space charge incoherent tune spread}

With bigh intensities, the proton beam particle distribution is Gaussian in transverse. This distribution yields large tune spread, which is betatron amplitude dependent. In general, the particle with small betatron amplitude has large tune shift, and vise versa. For bunched beams, also the particle at the azimuthal center has larger tüue shifi, etc.

On the other hand, the coherent tune, caused by the image effect and the broadband impedance, is in general depressed into the same direction of the incoherent tune shifts.

The space charge incoherent tune spread, therefore, is likely damping the microwave instabilities, especially for the strong instabilities. Due to the averaging effect, the rigid dipole motion and probably higher order mode motion with slow growth rate are less likely to be damped by this mechanism.

Several complications relevant to this damping mechanism are presented as follows.

1. The coherent tune shift is not always depressed. The examples include the image effect for non-circular chamber, and the broadband impedance effect under certain chamber geometries.

2. The complex particle distribution in the tune diagram implies large uncertainties in the damping mechanism.

3. Since there is no tune spread for the uniform distribution of the particle in phase space, the simulations using uniform distributions may be qualitatively misleading.

\section{AGS experience}

The transverse microwave instability is only presented at the high energy end of the AGS cycle, well above transition, at around $20 \mathrm{GeV}$. In Fig. 5A, the measured bunch length for the AGS cycle is shown, which is lengthened by the VHF cavity dilution in several spots in the cycle. At the injection, it helps to reduce the slow loss. Immediately above the transition, it is for longitudinal reasons. Only well above transition, from 0.8 second, the VHF dilution is necessary to suppress the transverse 
microwave instabilities. The bunch length according to the phase damping is also shown for comparison.

In Fig. 5B, it is shown that at the injection energy, the required tune spread, which equals to the space charge image and broadband impedance coherent tune shift, is much larger than the combined tune spread of the slippage, chromaticity, and the synchrotron tune. Yet, the machine is very stable in transverse. This might be explained by the large space charge incoherent tune spread at the injection, also shown in Fig. 5B.

At the high energy end, the space charge incoherent tune spread is reduced rapidly to comparable with the required tune spread. The transverse microwave instability may, therefore, develop.
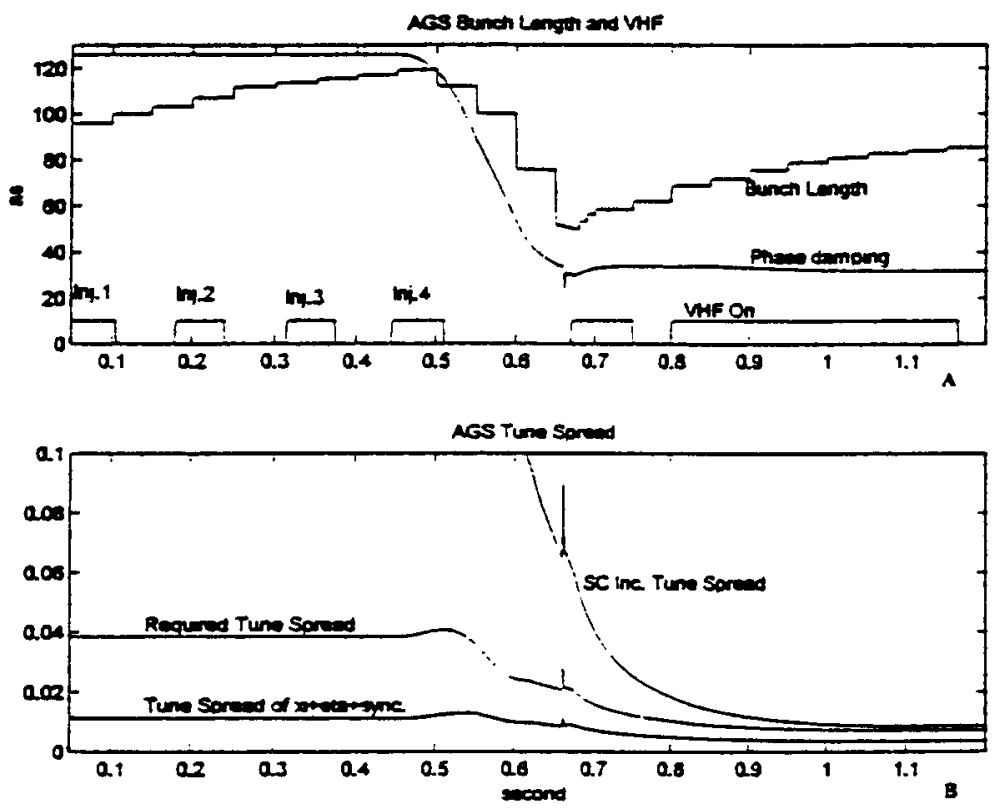

Fig. 5

\section{Conclusion}

Based on the observations of the low and medium energy proton synchrotrons, and the experiences at the AGS and the Booster, we may conclude that the transverse microwave instabilities will not take place at the SNS storage ring.

\section{PSR Instability}

The PSR type instability is quite different from the conventional transverse instabilities, and it is important to assure that the SNS storage ring will not be hampered by this type of instabilities. The study of the PSR instability at the BNL consists of several aspects. These include the analysis of the damping mechanism with respect 
to this type of instability, the issue of the gap clearing, and the secondary electron productions.

\section{A. The e-p instability}

The high proton beam potential at the PSR is blamed to attract and probably to accelerate electrons. Therefore, it is one of the main culprits of the e-p type oscillation. It is straightforward to calculate the potential well for the PSR, the SNS storage ring for $1 M W$ and $2 M W$ operations, as shown in Table 4.

\begin{tabular}{|l|l|l|l|l|}
\hline & PSR & SNS & SNS & \\
\hline$N$ & 4 & 10.4 & 20.8 & $10^{13}$ \\
\hline$R$ & 14.35 & 35.1 & 35.1 & $m$ \\
\hline$B_{f}$ & 0.4 & 0.4 & 0.4 & \\
\hline$b$ & 5 & 10 & 10 & $\mathrm{~cm}$ \\
\hline$a=\sqrt{2} \sigma$ & 1.2 & 2.4 & 2.4 & $\mathrm{~cm}$ \\
\hline$V_{\text {pot. }}$ & 6 & 6.5 & 13 & $\mathrm{KV}$ \\
\hline
\end{tabular}

Table 4. Potential well

It is shown that the potential well of the $1 M \Gamma W$ SNS storage ring is about the same as the PSR. For $2 M W$ SNS, it is about twice as high.

One also notice that for ep type instability, the space charge incoherent tune spread is not effective in damping. The reason is as follows.

1. The electron induced proton beam coherent tune shift is not depressed as the image effect or broadband impedance effect. Instead, with the electrons in the proton beam, the coherent tune tends to move out of the space charge incoherent tune spread, in the tune diagram.

2. If multipacting takes place, the neutralization factor could be large. At the SNS, the neutralization factor of $\eta_{\text {reu }}=0.23$ could entirely offset the space charge effect, leading to zero tune spread.

These results have shown the existence of the mechanism of the e-p instability at the SNS storage ring. However, with the same importance, the damping mechanism needs also to be studied by comparing the two machines.

\section{B. Damping mechanism of momentum spread}

Numerous evidences at the PSR have showed that the beam momentum spread is important in damping the e-p instability. 
1. At the PSR, threshold is proportional to $R F$ voltage $V_{R F}$. From the relation [21],

$$
N \propto n \Delta p / p \propto \sqrt{N} \sqrt{V_{R F}}
$$

where $n$ is the electron bouncing mode number, we get

$$
N \propto V_{R F}
$$

This implies also

$$
N \propto(\Delta p / p)^{2}
$$

2. Instability improved by inserting ferrite rings in the beam pipe, which cleared the gap, but also increased beam momentum spread.

3. Coasting beam threshold increases with the larger Linac beam momentum spread.

4. Significant chromatic effect has been recently shown in the study, which is re lated with the beam momentum spread.

5. Double RF study showed no change on the threshold. The peak current is reduced applying the second barmonic $R F$, but the beam momentum spread also reduced. Two effects may offset.

6. Increasing the bare tune by one unit improved the instability. It was explained by the effect of $\xi \nu_{0} \Delta p / p$.

The typical beam momentum spread at the SNS is about twice as much as the PSR. Also, for $2 M W$ SNS, the electron bouncing frequency is about twice as high as the PSR. Given the similar slippage factor, the tune spread at the $2 M W$ SNS is about 4 times as large as the PSR. These are shown in Table 5 . Inserting the negative impact of the $2 M W$ SNS high potential well, the factor of $\Delta \nu / V_{\text {pot. }}$ at the $2 M W$ SNS is still about twice as high as the PSR. For the $1 M N W$ SNS, this factor is about 3 times in favor of the SNS.

\begin{tabular}{|l|l|l|l|l|}
\hline & PSR & SNS, 1MW & SNS, 2MW & \\
\hline$\Delta p / p$ & 0.34 & 0.7 & 0.7 & $\%$ \\
\hline$n$ & 60 & 86 & 120 & \\
\hline$\eta$ & -0.188 & -0.193 & -0.193 & \\
\hline$\Delta \nu=n \eta \Delta p / p$ & 3.8 & 11.6 & 16.2 & $\%$ \\
\hline$\Delta \nu / V_{\text {pot }}$ & 0.63 & 1.78 & 1.25 & \\
\hline
\end{tabular}

Table 5: Comparison of Tune Spread due to $\Delta p / p$

It is, therefore, possible that the e-p instability will not be seen at the SNS. Efforts at the SNS, however, go on for better understanding of the e-p type instability, damping mechanism, and other studies. 


\section{Other studies}

Other studies toward understanding and preventing the e-p type instability at the SNS are summarized as follows.

1. Gap clearing

- As mentioned previously, in addition to the high RF voltage used in the stacking, a ramping of RF voltage from $20 \mathrm{KV}$ to $40 \mathrm{KV}$ at the end of stacking is proposed [22].

- A gap cleaning kicker is proposed [23].

- The effect of longitudinal impedance of the extraction kickers is under study.

2. Secondary electron (SE) production

- The projectile scraping effect in SE production is verified in crashing the gold beam into the Booster injection septum. Translated equivalent SNS proton SE production rate was about 27 [24].

- A systematic study of this effect at the BNL Tandem van de Graaff has been performed, using gold, oxygen, and proton ions. The scraping effect of $1 / \cos \theta^{-1.15}$ has been obtained [25].

- Also in the Tandem study, it has been shown that using the serrated surface for the collimator, the SE yield is reduced by more than a factor of 10 [25].

- Further studies for different surface, coatings, conditioned surface are under planning.

- Possible multipacting effect for the proton beams in the PSR and SNS is under studying [26]. 


\section{Research Bibliography}

${ }^{1}$ W.T. Weng, AIP Conference Proceedings, 448, Shelter Island, New York, 1998.

${ }^{2} \mathrm{R}$. Macek, ibid.

${ }^{3}$ R.E. Shafer, IEEE Trans. NS, Vol.NS-32, No.5, p.1933, 1985.

${ }^{4}$ K.Y. Ng, Particle Accelerators, Vol.23, p.93, 1988.

${ }^{5}$ S.S. Kurennoy and G.V. Stupakov, Particle Accel, Vol.45, p.95, 1994.

${ }^{6}$ D. Boussard and J. Gareyte, SPS/AC, Impr. Report No.181, CERN 1980.

${ }^{7}$ T. Linnecar and E. Shaposhnikova, CERN-SL-96-45, RF, July, 1996.

${ }^{8}$ F. Pedersen and E. Raka, IEEE Trans. NS, Vol.NS-26, No.3, p.3592, 1979.

${ }^{9} \mathrm{R}$. Cappi, these proceedings.

${ }^{10}$ A. Chao, Physics of Collective Beam Instabilities in High Energy Accelerators, Wiley, New York, 1994.

${ }^{11}$ G. Nassibian and F. Sacherer, Nucl. Inst. Meth. Vol.159, p.21, 1979.

${ }^{12}$ G. Lambertson, Workshop on RHIC Performance, March, 1988.

${ }^{13}$ W.Z. Meng, private communication.

${ }^{14} \mathrm{~J}$. Griffin et. al., FN-661, 1997.

${ }^{15}$ K. Koba, et. al., KEK Preprint 97-173, 1997.

${ }^{16} \mathrm{~K} . Y . \mathrm{Ng}$, Fermilab-FN-659, July 7, 1997.

${ }^{17}$ W.K. vanAsselt and Y.Y. Lee, Proc. of Part. Accel. Conf., p.881, 1991.

${ }^{18}$ F. Voelker and G. Lambertson, Proc. of Part. Accel. Conf., p.851, 1989.

${ }^{19}$ D. Neuffer et al, Nucl. Instr. and Meth. A321, p.1, 1992.

${ }^{20} \mathrm{~J}$. Gareyte, Frontier of Particle Beams: Intensity Limitations, Springer-Verlag, New York, 1990.

${ }^{21} \mathrm{R}$. Macek, these proceedings.

${ }^{22}$ S.Y. Zhang and W.T. Weng, Proc. of Euro. Part. Accel. Conf., p.1139, 1998, Stockbolm, Sweden.

${ }^{23} \mathrm{~J}$. Wei, private communication.

${ }^{24}$ S.Y. Zhang, Proc. of Part. Accel. Conf., p.3297, 1999, New York, New York.

${ }^{25} \mathrm{P}$. Thieberger et. al., in preparation.

${ }^{26}$ M. Blaskiewicz, Proc. of Part. Accel. Conf., p.1611, 1999, New York, New York. 


\section{.}

4 